\title{
Twist periodic orbits and topological entropy for continuous maps of the circle of degree one which have a fixed point
}

\author{
LLUÍS ALSEDÀ ${ }^{1,2}$, JAUME LLIBRE ${ }^{1}$, MICHAL MISIUREWICZ \\ AND CARLES SIMÓ
}

' Seccio de Matemàtiques, Facultat de Ciències, Universitat Autònoma de Barcelona, Bellaterra, Barcelona, Spain; ${ }^{2}$ Departament de Teoria Econòmica, Facultat de Ciències Econòmiques, Universitat Autònoma de Barcelona, Bellaterra, Barcelona, Spain; ${ }^{3}$ Instytut Matematyki, Uniwersytet Warszawski, Pałac Kultury i Nauki IX p., 00-901 Warszawa, Poland ; ${ }^{4}$ Facultat de Matemàtiques, Universitat de Barcelona, Barcelona, Spain

(Received 14 February 1984 and revised 28 February 1985)

Abstract. Let $f$ be a continuous map from the circle into itself of degree one, having a periodic orbit of rotation number $p / q \neq 0$. If $(p, q)=1$ then we prove that $f$ has a twist periodic orbit of period $q$ and rotation number $p / q$ (i.e. a periodic orbit which behaves as a rotation of the circle with angle $2 \pi p / q$ ). Also, for this map we give the best lower bound of the topological entropy as a function of the rotation interval if one of the endpoints of the interval is an integer.

\section{Introduction and results}

Let $S^{1}$ be the circle. We denote by $C_{1}\left(S^{1}\right)$ the set of all continuous maps from $S^{1}$ to itself of degree one. For $x \in S^{1}$, we say that $x$ is periodic if there exists a positive integer $n$ such that $f^{n}(x)=x$. The period of $x$ is the smallest integer satisfying this relation. Let $P(f)$ be the set of periods of $f$. If $x \in S^{1}$ is a periodic point of period $n$, then the orbit of $x$ is the set $\left\{f^{k}(x): k=1,2, \ldots, n\right\}$. We refer to such an orbit as a periodic orbit of period $n$.

Let $f \in C_{1}\left(S^{1}\right), F$ its lifting to the covering space $\mathbb{R}$ and $e(X)=\exp (2 \pi i X)$ the natural projection of $\mathbb{R} \rightarrow S^{1}$. We note that $F$ is not defined uniquely; nevertheless, if $F$ and $F^{\prime}$ are two liftings of $f$ then $F=F^{\prime}+m$ with $m \in \mathbb{Z}$. Since $\operatorname{deg}(f)=1$ we have $F(X+1)=F(X)+1$ for all $X \in \mathbb{R}$. If $x$ is a periodic point of $f$ of period $n$ and $e(X)=x$, then $F^{n}(X)=X+k$ where $k \in \mathbb{Z}$. We shall call $k / n$ the rotation number (or $F$-rotation number, if necessary) of $x$ and we denote it by $\rho(x)$ or $\rho_{F}(x)$. We denote by $L(f)$ or $L_{F}(f)$ the set of all rotation numbers of $f$. The following statements are known (see [2] and [5]):

(1) $\rho(x)$ does not depend on the choice of $X$. Actually, it depends on the periodic orbit. 
(2) If $F=F^{\prime}+m$ then $\rho_{F}(x)=\rho_{F}(x)+m$.

(3) $\rho_{F^{m}}(x)=m \rho_{F}(x)$.

(4) If $a<k / n<c, a, c \in L(f)$ and $k, n \in \mathbb{Z}$ with $n>0$, then $k / n \in L(f)$ and $n \in P(f)$.

(5) $L(f) \cap \mathbb{Z} \neq \varnothing$ if and only if $1 \in P(f)$.

(6) If $a_{m} \in L(f)$ for $m=1,2, \ldots$ and $a=\lim _{n \rightarrow \infty} a_{m} \in \mathbb{Q}$, then $a \in L(f)$.

(7) If $f$ has no periodic points, then $\lim _{n \rightarrow \infty}\left(F^{n}(X)-X\right) / n$ exists for all $X$, it is independent of $X$ and is irrational.

From the above statements, we can write $L(f)=[a, b] \cap \mathbb{Q}$ for some $a, b \in \mathbb{R}$. That is, $L(f)$ is a closed interval (possibly degenerated to one point) on $\mathbb{Q}$ and, from now on, we shall call it the rotation interval of $f$. If $f$ has no periodic points, one can take $a=b=\lim _{n \rightarrow \infty}\left(F^{n}(X)-X\right) / n$.

From now on for a rational number $p / q$ we always assume $q>0$. Let $f \in C_{1}\left(S^{1}\right)$ and $W$ be a periodic orbit of $f$ of period $q$ and rotation number $p / q$ with $(p, q)=1$. Let $F$ be a lifting of $f$ such that $p / q \in L_{F}(f)$. Suppose that $F$ is order preserving on the set $e^{-1}(W)$. Then we say that $W$ is a twist periodic orbit (from now on TPO) of $f$ of period $q$ and rotation number $p / q$. Notice that every periodic orbit of period 1 is a TPO.

Let $x, y \in S^{1}$. We denote by $(x, y)$ (resp. $\left.[x, y]\right)$ the open (resp. closed) arc of $S^{1}$ from $x$ counterclockwise to $y$. Let $W=\left\{x_{1}, \ldots, x_{q}\right\}$ be a periodic orbit of $f$ of period $q$ and rotation number $p / q$ with $(p, q)=1$. Suppose that either $\left(x_{i}, x_{i+1}\right) \cap W=\varnothing$ for $i=1,2, \ldots, q-1$ and $\left(x_{q}, x_{1}\right) \cap W=\varnothing$ if $p / q>0$, or $\left(x_{i+1}, x_{i}\right) \cap W=\varnothing$ for $i=$ $1,2, \ldots, q-1$ and $\left(x_{1}, x_{q}\right) \cap W=\varnothing$ if $p / q<0$. To give a geometrical interpretation of a TPO on the circle, we shall prove that if $W$ is a TPO then

$$
f\left(x_{i}\right)=x_{i+|p|(\bmod q)} \quad \text { for } i=1,2, \ldots, q
$$

and the converse is not true (see lemma 1 ). Note that the notion of TPO of period $q$ and rotation number $p / q$ characterizes the simplest behaviour of the graph of a map which has this rotation number.

THEOREM A. Let $f \in C_{1}\left(S^{1}\right)$. Then the following hold:

(a) If $r / s$, with $(r, s)=1$, is an endpoint of $L(f)$, then all periodic orbits of $f$ of period $s$ and rotation number $r / s$ are TPO.

(b) If $p / q \in L(f)$ with $(p, q)=1$, then there exists a TPO of $f$ of period $q$ and rotation number $p / q$.

In the first version of this paper, we assumed in theorem $\mathrm{A}$ (b) that also $0 \in L(f)$. Then this result was generalized by one of the authors (see [6]). The proof given here is much simpler than the one from [6]; (this theorem has been proved (in another simple way) independently by A. Chenciner, J.-M. Gambaudo and Ch. Tresser (C.R. Acad. Sci. Paris, t. 299, Sér. I (1984), 145-148). It is based on the methods used by $R$. Hall for the case of twist maps of the annulus.

From now on, $E(\cdot)$ will denote the integer part function. Let $c \in \mathbb{R}, c \neq 0$. For $z>1$ we define

$$
Q_{c}(z)=z+1-2 \sum_{n=0}^{\infty} z^{-E(n /|c|)}
$$


Let $\beta_{c}$ be the largest zero of the equation $Q_{c}(z)=0$. If $f \in C_{1}\left(S^{1}\right)$ then $h(f)$ denotes the topological entropy of $f$.

THEOREM B. Let $f \in C_{1}\left(S^{1}\right)$ and suppose that $L(f)$ contains either $[0, c] \cap \mathbb{Q}$ or $[c, 0] \cap \mathbb{Q}$ with $c \neq 0$. Then $h(f) \geq \log \beta_{c}$ Moreover, for every $c \in \mathbb{Q}, c \neq 0$ there exists a map $f \in C_{1}\left(S^{1}\right)$ such that $L(f)$ is either $[0, c] \cap \mathbb{Q}$ or $[c, 0] \cap \mathbb{Q}$ and $h(f)=\log \beta_{c}$.

For a rational number $p / q \neq 0$ such that $(p, q)=1, q>0$, we define the following polynomial

$$
H_{p / q}(z)=z^{q+1}-z^{q}-z-1-2 \sum_{j=1}^{|p|-1} z^{E(j q /|p|)+1} .
$$

We denote by $\alpha_{p / q}$ the largest root of $H_{p / q}$

THEOREM C. The following statements hold:

(a) For $c \neq 0$ the function $Q_{c}$ has a unique root (larger than 1).

(b) For $p / q \neq 0$ and $(p, q)=1$ we have $H_{p / q}(z)=\left(z^{q}-1\right) Q_{p / q}(z)$. Then $\alpha_{p / q}=\beta_{p / q}$.

(c) The function $\beta .: \mathbb{R} \backslash\{0\} \rightarrow(1, \infty)$ is strictly increasing on the positive numbers. Moreover $\beta_{-c}=\beta_{c}$. Hence it is strictly decreasing on the negative numbers.

(d) The function $\beta$. is continuous from the left at every $c>0$ (and from the right at every $c<0$ ).

(e) The function $\beta$. is continuous at all irrational points.

(f) The function $\beta$. is discontinuous from the right at all positive rational numbers (and from the left at all negative rational numbers).

(g) For every $n \in \mathbb{N}$, the jump of $\beta$. occurring at $n$ is $1+\sqrt{n^{2}+2 n}-\sqrt{n^{2}+1}$ which belongs to the interval $(2-1 / n, 2-1 /(n+1))$; the sum of all other jumps occurring between $n$ and $n+1$ is $2 /\left(\sqrt{n^{2}+2 n}+\sqrt{n^{2}+2 n+2}\right)<1 / n$.

Theorems $\mathrm{A}, \mathrm{B}$, and $\mathrm{C}$ are proved in $\$ \S 3,4$.

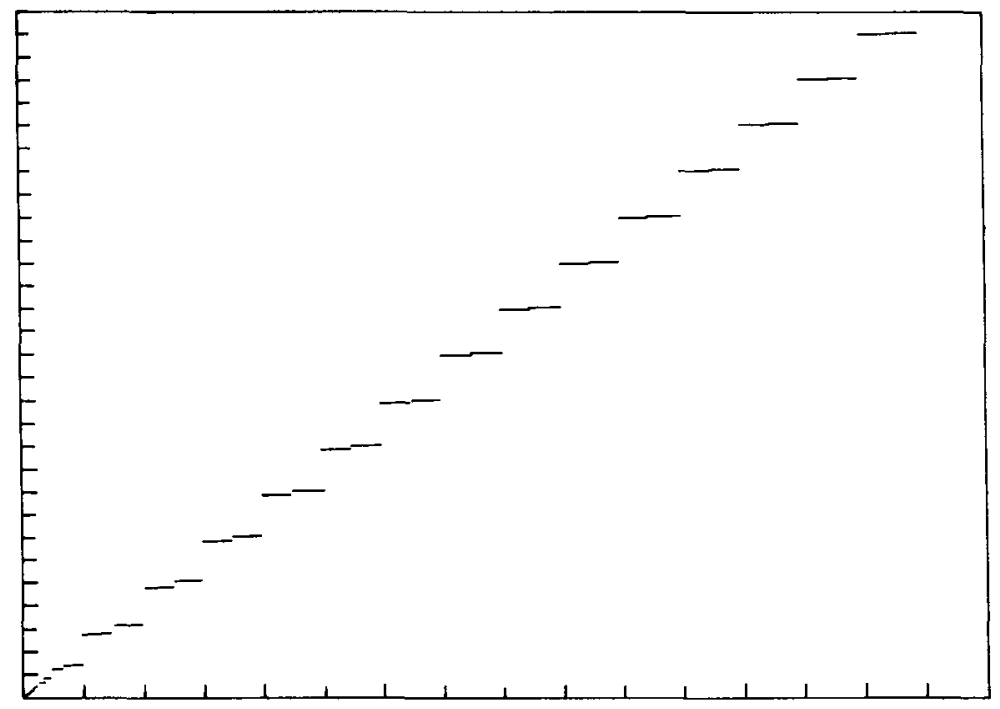

FIGURE 1(a) 


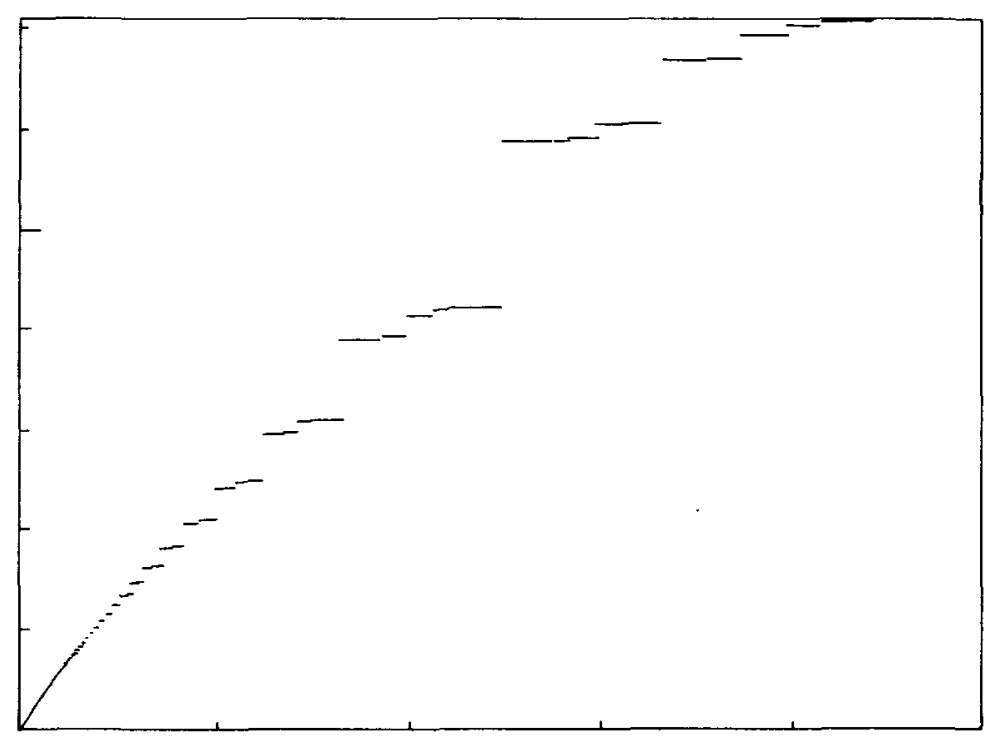

FigURE 1(b)

FIGURE 1. This shows the graph of the map $\beta$. In (a) $c$ lies in $[0,16], \beta$ in $[1,16+\sqrt{257}]$. In (b) $c$ lies in $[0,1], \beta$ in $[1,2+\sqrt{5}]$. Each large division is equal to one unit.

Remark 1. In [5] the following result is shown. Let $f \in C_{1}\left(S^{1}\right)$ and $L(f)=[a, b] \cap \mathbb{Q}$. Then, there exist $s_{1}, s_{2} \in \mathbb{N} \cup\left\{2^{\infty}\right\}$ such that $P(f)=S\left(a, s_{1}\right) \cup M(a, b) \cup S\left(b, s_{2}\right)$, where

$$
\begin{aligned}
& M(a, b)=\{n \in \mathbb{N}: a<k / n<b \text { for some } k \in \mathbb{Z}\}, \\
& S\left(a, s_{1}\right)=\left\{\begin{aligned}
\varnothing, \quad \text { if } a \notin \mathbb{Q} ; \\
\left\{n \cdot 2^{m}: m \in \mathbb{N} \cup\{0\} \text { and } a=k / n \text { with }(k, n)=1\right\}, \\
\text { if } a \in \mathbb{Q} \text { and } s_{1}=2^{\infty} ; \\
\{n \cdot s: a=k / n \text { with }(k, n)=1 \text { and } s \text { is larger than } \\
\text { or equal to } \left.s_{1} \text { in the Sarkovskii's ordering }\right\}, \\
\text { if } a \in \mathbb{Q} \text { and } s_{1} \neq 2^{\infty} .
\end{aligned}\right.
\end{aligned}
$$

$S\left(b, s_{2}\right)$ is as $S\left(a, s_{1}\right)$ with $b$ and $s_{2}$ instead of $a$ and $s_{1}$, respectively. Moreover, for every set $A \subset \mathbb{N}$ of the above form, there exists a continuous map $f \in C_{1}\left(S^{1}\right)$ with the corresponding rotation interval and $P(f)=A$.

From this result it is easy to see that if $a \in \mathbb{Z}$ or $b \in \mathbb{Z}$ then $P(f)=\mathbb{N}$ if $b-a \geq \frac{1}{2}$. Moreover, suppose that $b-a \in[1 / n, 1 /(n-1))$ with $n \geq 2$. Then

$$
M(a, b) \cup S\left(b, s_{2}\right)=\{n, n+1, \ldots\} \quad \text { if } a \in \mathbb{Z},
$$

and

$$
M(a, b) \cup S\left(a, s_{1}\right)=\{n, n+1, \ldots\} \quad \text { if } b \in \mathbb{Z} .
$$

Clearly, this implies that there are a lot of maps of the circle of degree one with different rotation interval and the same set of periods. Thus, for $f \in C_{1}\left(S^{1}\right)$ the study of lower bounds of the topological entropy must be more precise using the rotation interval than using the set of periods $P(f)$. 
Remark 2. Theorem B gives us the best lower bound of the topological entropy of a map $f \in C_{1}\left(S^{1}\right)$ depending on its rotation interval if one of the endpoints of the interval is an integer. Now we improve this lower bound taking into account the set of periods due to the endpoints of the rotation interval. That is, using the notation of remark 1 and taking $a=0$, from theorem 3.2 of [2] we have that

$$
h(f) \geq\left(\log \lambda_{q}\right) / 2^{m}
$$

if $s_{1}=2^{m} q$ with $q \geq 3$ odd, where $\lambda_{q}$ is the largest root of the polynomial $z^{q}-2 z^{q-2}-1$. Then we have

$$
h(f) \geq \max \left\{\left(\log \lambda_{q}\right) / 2^{m}, \log \beta_{c}\right\},
$$

where either $s_{1}=2^{m} q, c=b$ if $a=0$, or $s_{2}=2^{m} q, c=a$ if $b=0$, and $q \geq 3$ is odd. Moreover, if $\{a, b\} \cap \mathbb{Z} \neq \varnothing$, then it is easy to see (from theorem $B$ of [1], [4] and statement (c) of theorem $C$ ) that

$$
\left(\lambda_{q}\right)^{2^{-m}} \leq \lambda_{3} \leq \beta_{c} \quad \text { if } b-a \geq \frac{1}{3} .
$$

Therefore, if $b-a \geq \frac{1}{3}$ and $\{a, b\} \cap \mathbb{Z} \neq \varnothing$, then we only need theorem B to give good lower bounds of the topological entropy.

Remark 3. From [5] we have that if $f \in C_{1}\left(S^{1}\right)$ and $0 \in \operatorname{Int} L(f)$ then $h(f) \geq \log 3$. On the other hand, from lemma 23 we have that, if $|c| \searrow 0$ (resp. $|c| \nearrow n,|c| \searrow n$ where $n \in \mathbb{N}$ ) we have $\beta_{c} \searrow 1$ (resp. $\left.\beta_{c} \nearrow n+\left(n^{2}+1\right)^{\frac{1}{2}}, \beta_{c} \searrow n+1+\left[(n+1)^{2}-1\right]^{\frac{1}{2}}\right)$. Therefore, theorem $B$ gives better lower bounds of the topological entropy in the case that $L(f)$ is of one of the following types:

(a) $[0, c]$ with $0<c \leq 1$;

(b) $[c, 0]$ with $-1 \leq c<0$; or

(c) $[a, b]$ such that $-1<a \leq 0$, and $b>1$.

\section{Preliminary results}

LEMMA 1. Let $f \in C_{1}\left(S^{1}\right)$ and let $T=\left\{x_{1}, x_{2}, \ldots, x_{q}\right\}$ be a TPO of period $q$ and rotation number $p / q$. Suppose that either $\left(x_{i}, x_{i+1}\right) \cap T=\varnothing$ for $i=1,2, \ldots, q-1$ and $\left(x_{q}, x_{1}\right) \cap$ $T=\varnothing$ if $p / q>0$, or $\left(x_{i+1}, x_{i}\right) \cap T=\varnothing$ for $i=1,2, \ldots, q-1$ and $\left(x_{1}, x_{q}\right) \cap T=\varnothing$ if $p / q<0$. Then we have that $f\left(x_{i}\right)=x_{i+|p|(\bmod q)}$ for $i=1,2, \ldots, q$, and $(p, q)=1$. Moreover, the converse is not true, i.e. the last assertions do not imply that the periodic orbit is twist.

Proof. We suppose that $p / q>0$. For the case $p / q<0$ the proof is analogous. Let $F$ be the lifting of $f$ for which $\rho_{F}\left(x_{1}\right)=p / q$. We have $e^{-1}(T)=\left\{X_{i}: i \in \mathbb{Z}\right\}$, with $\cdots \mathrm{X}_{-2}<\mathrm{X}_{-1}<\mathrm{X}_{0}<\mathrm{X}_{1}<\mathrm{X}_{2} \cdots$. We may assume that $e\left(X_{1}\right)=x_{1}$. Then, clearly we have $X_{i+k q}=X_{i}+k$ and $e\left(X_{i+k q}\right)=x_{i}$ for $k \in \mathbb{Z}$ and $i=1,2, \ldots, q$. Since $F$ on $e^{-1}(T)$ is one-to-one and order preserving, we have $F\left(X_{i}\right)=X_{i+v}$ for some $v \in \mathbb{Z}$ and all $i$. But

$$
X_{i}+p=F^{q}\left(X_{i}\right)=X_{i+v q}=X_{i}+v
$$

and therefore $v=p$, and $f\left(x_{i}\right)=x_{i+p(\bmod q)}$ for all $i$. Since $T$ is an orbit (not a union of several orbits) we obtain $(p, q)=1$.

To see that the converse is not true, look at figure 2 for an example with $p / q=2 / 5$. 


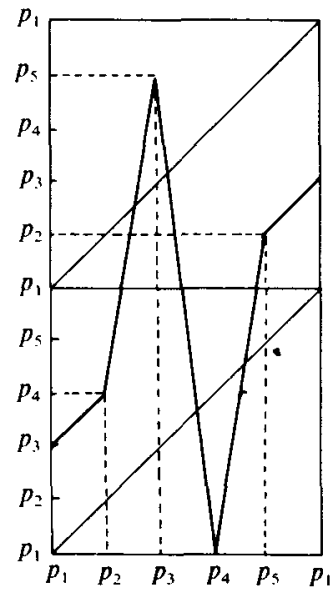

FIGURE 2

From now on we assume that the notions and techniques of [2] are known to the reader.

LeMMA 2 (see [2]). Let $f \in C_{1}\left(S^{1}\right)$. If $J_{0} \rightarrow J_{1} \rightarrow \cdots \rightarrow J_{n-1} \rightarrow J_{0}$ is a loop in an A-graph of $f$, then there exists a fixed point $x$ of $f^{n}$ such that $f^{i}(x) \in J_{i}$ for $i=0,1, \ldots, n-1$.

Let $f \in C_{1}\left(S^{1}\right)$ and $A=\left\{a_{1}, \ldots, a_{n}\right\}$ be an invariant set (i.e. $\left.f(A) \subset A\right)$. Let $X_{1}<X_{2}<$ $\cdots<X_{n}<X_{1}+1$ be such that $e\left(X_{i}\right)=a_{i}$ for $i=1,2, \ldots, n$. Let $F$ be a lifting of $f$. We denote by $\bar{F}$ the map such that

(1) $\bar{F}\left(X_{i}+k\right)=F\left(X_{i}+k\right)$ for $i=1,2, \ldots, n$ and $k \in \mathbb{Z}$;

(2) $\left.\bar{F}\right|_{\left[X_{i}+k, X_{i+1}+k\right]}$ is linear for $i=1,2, \ldots, n-1$ and $k \in \mathbb{Z}$;

(3) $\left.\bar{F}\right|_{\left[X_{n}+k, X_{1}+k+1\right]}$ is linear for $k \in \mathbb{Z}$.

We call $\bar{F}$ the $A$-linearization of $F$. We denote by $\bar{f}$ the map of the circle of degree one which has $\bar{F}$ as a lifting. We say also that $\bar{f}$ is the A-linearization of $f$. If $\bar{F}=F$, that is, $\bar{f}=f$, then we say that $F$ and $f$ are $A$-linear.

LEMMA 3. Let $g$ be an increasing map (not necessarily continuous) of a closed interval $I$ into itself. Then $g$ has a fixed point.

Proof. Take $X=\sup \{Y: g(Y)>Y\}$. If $g(X)>X$ then for every $Y \in(X, g(X))$ we have $g(Y)>g(X)>Y$ and this is a contradiction. If $g(X)<X$ then for every $Y \in(g(X), X)$ we have $g(Y)<g(X)<Y$, again a contradiction. Therefore, $g(X)=X$.

Let $I$ be an interval of $\mathbb{R}$. We denote by $I+n$ the set $\{X+n: X \in I\}$.

LEMMA 4. Let $f \in C_{1}\left(S^{1}\right)$ with lifting $F$ and let $A$ be a finite invariant set under $f$. Let $\bar{f}$ be the A-linearization of $f$ and suppose that $\bar{f}$ has a TPO Tof period s and $\bar{F}$-rotation number $r / s$ with $(r, s)=1$, where $\bar{F}$ is the lifting of $\bar{f}$ obtained by A-linearization of $F$. If $T \not \subset A$ and $\bar{F}$ is increasing at every point of $e^{-1}(T)$, then $f$ has a TPO of period $s$ and $F$-rotation number $r / s$. 
Proof. Let $X_{1}<X_{2}<\cdots<X_{n}<X_{1}+1$ be such that $e\left(\left\{X_{1}, X_{2}, \ldots, X_{n}\right\}\right)=A, I_{i}=$ $\left[X_{i}, X_{i+1}\right]$ for $i=1,2, \ldots, n-1$ and $I_{n}=\left[X_{n}, X_{1}+1\right]$. Let $U$ be the partition of $\mathbb{R}$ given by the points of $e^{-1}(A)$. Since $T \not \subset A$ is a periodic orbit and $A$ is invariant for $\bar{f}$, they are disjoint. Hence, if we choose $x \in T$, then there is a unique $i_{1} \in\{1, \ldots, n\}$ such that there exists $X \in I_{i_{1}}$ with $e(X)=x$. Analogously, there are unique $i_{j} \in$ $\{1, \ldots, n\}$ and $n_{j} \in \mathbb{Z}$ such that $\bar{F}^{j-1}(X) \in I_{i_{j}}+n_{j}, j=2, \ldots, s+1$. Since $\bar{F}^{s}(X)=X+r$, we have $i_{s+1}=i_{1}$ and $n_{s+1}=r$. In such a way we obtain the following path in the $U$-graph of $\bar{F}$ :

$$
I_{i_{1}} \rightarrow I_{i_{2}}+n_{2} \rightarrow \cdots \rightarrow I_{i_{s}}+n_{s} \rightarrow I_{i_{1}}+r .
$$

Now we define the following maps (perhaps non-continuous):

$$
\begin{aligned}
& M_{s}: I_{i_{1}}+r \rightarrow I_{i_{s}}+n_{s}, \\
& M_{j}: I_{i_{j+1}}+n_{j+1} \rightarrow I_{i_{j}}+n_{j} \quad \text { for } j=1,2, \ldots, s-1,
\end{aligned}
$$

such that $M_{j}(X)=\inf \left\{Y \in I_{i_{j}}+n_{j}: F(Y)=X\right\}$. Since $\bar{F}$ is increasing on every interval $I_{i_{j}}+n_{j}, j=1,2, \ldots, s$, these maps are increasing (see, for instance, figure 3 , where $\left.Y_{i}=M_{j}\left(Z_{i}\right), \quad i=1,2,3\right)$. Therefore, the map $M: I_{i_{1}}+r \rightarrow I_{i_{1}}+r$ such that $M=$ $M_{1} \circ M_{2} \circ \cdots \circ M_{s}+r$ is increasing. By lemma $3, M$ has a fixed point $Z^{\prime} \in I_{i_{1}}+r$. Let $Z=Z^{\prime}-r \in I_{i_{1}}$. Obviously, $F^{j}(Z) \in I_{i_{j+1}}+n_{j+1}$ for $j=0,1, \ldots, s-1$, and $F^{s}(Z)=$ $Z+r \in I_{i_{1}}+r$. Therefore $\left\{e(Z), f(e(Z)), \ldots, f^{s-1}(e(Z))\right\}$ is a periodic orbit of period $s$ and rotation number $r / s$. To see that it is a TPO, one has to look at $\left\{F^{j}(Z): j=\right.$ $0, \ldots, s-1\}+\mathbb{Z}$ and compare it with $e^{-1}(T)$. To decide which of two given points of such a set is to the left and which to the right, one has to check first to which elements of the $U$-partition they do belong. If these intervals are different, then the answer is obvious. If they are the same, then one has to go along the orbits of these two points and keep checking. Since we only use increasing pieces of the maps $F$ and $\bar{F}$, we have that the procedure and criteria are the same in both cases (i.e. in the case of $\left\{F^{j}(Z): j=0,1, \ldots, s-1\right\}+\mathbb{Z}$ and the case of $\left.e^{-1}(T)\right)$, and the ordering of these two sets is the same. Hence, since $T$ is a TPO, so is $\left\{e(Z), f(e(Z)), \ldots, f^{s-1}(e(Z))\right\}$.

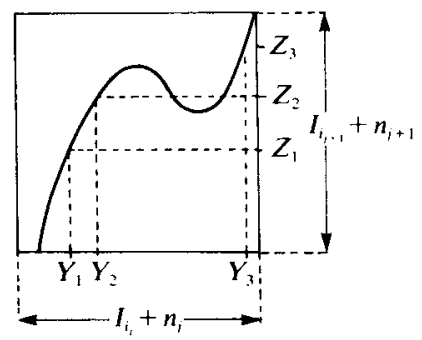

FIGURE 3

\section{Twist periodic orbits}

Let $f \in C_{1}\left(S^{1}\right)$ and $F$ be a lifting of $f$. We define the maps $F_{r}$ and $F_{l}$ as follows (see figure 4):

$$
\begin{aligned}
& F_{r}(X)=\sup \{F(Y): Y \leq X\} \\
& F_{l}(X)=\inf \{F(Y): Y \geq X\}
\end{aligned}
$$


We write $f_{r}$ and $f_{l}$ for the maps of the circle which have as a lifting $F_{r}$ and $F_{l}$, respectively. Obviously $f_{n} f_{l} \in C_{1}\left(S^{1}\right)$.

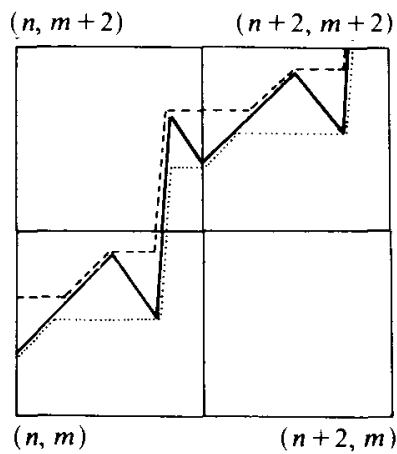

FIGURE 4. - - denotes $F_{r} ;-$ denotes $F ; \cdots$ denotes $F_{r}$.

LeMmA 5. Let $f \in C_{1}\left(S^{1}\right)$ and let $W$ be a periodic orbit of $f$ of period $q$ and rotation number $p / q$ such that $0<p / q<1$ and $(p, q)=1$. Suppose that $W$ is not a TPO. Then the following hold:

(a) fhas a TPO $R$ of period $s$ and rotation number $r / s$ with $(r, s)=1$ and $p / q<r / s$.

(b) $f$ has a TPO $L$ of period $n$ and rotation number $m / n$ with $(m, n)=1$ and $m / n<p / q$.

Proof. We prove (a) and (b) in the case when $f$ is $W$-linear. Also, in this case, we prove that $F$ is increasing on $e^{-1}(R)$ and $e^{-1}(L)$. Then we may use lemma 4 and we obtain (a) and (b) in the general case. We deal with statement (a); the case (b) is similar.

Let $U$ be the partition of $S^{1}$ by the elements of $W$. Since $f_{r}$ is onto, for every $I \in U$ there exists $J \in U$ such that $J f_{r}$-covers $I$. Therefore, since the number of intervals of $U$ is finite, we have a loop of length $s$ in the $U$-graph of $f_{r}$ with $1 \leq s \leq q$. In addition we assume that this loop is the shortest one of the $U$-graph of $f_{r}$ Moreover, since $W$ is not TPO at least one interval $I \in U$ satisfies $\left.f_{r}\right|_{I}=$ constant. Then $s<q$. From lemma 2, this loop gives us a periodic orbit $R$ of $f_{r}$ of period $s$ and rotation number $r / s$. Since $s<q$, we have $R \neq W$. All intervals on which $F_{r}$ is constant are mapped to elements of $W$, and hence $F_{r}$ is increasing at every point of $e^{-1}(R)$. Consequently $\left.F\right|_{e^{-1}(R)}=\left.F_{r}\right|_{e^{-1}(R)}$. Since $F_{r}$ is non-decreasing, $R$ is a TPO for $f_{r}$ Hence, it is a TPO for $f$. From lemma 1 it follows that $(r, s)=1$.

To finish the proof of (a) we only need to show that $r / s \geq p / q$. Since $F_{r}$ is non-decreasing and $F_{r} \geq F$, we have $F_{r}^{n} \geq F^{n}$ for all $n$. Hence, if $X \in e^{-1}(R), Y \in$ $e^{-1}(W)$ and $X>Y$, then

$$
\begin{aligned}
r / s & =\lim _{n \rightarrow \infty}\left(F_{r}^{n}(X)-X\right) / n=\lim _{n \rightarrow \infty} F_{r}^{n}(X) / n \geq \lim _{n \rightarrow \infty} F_{r}^{n}(Y) / n \\
& \geq \lim _{n \rightarrow \infty} F^{n}(Y) / n=\lim _{n \rightarrow \infty}\left(F^{n}(Y)-Y\right) / n=p / q .
\end{aligned}
$$

Now we consider the space $\mathscr{L}$ of all liftings of maps from $C_{1}\left(S^{1}\right)$, with the topology of uniform convergence. The maps $F, G, F_{t}, G_{n}$ will be liftings of the maps $f, g, f_{t}$, 
$g_{n}$ respectively. We fix $p \in \mathbb{Z}$ and $q \in \mathbb{Z}^{+}$such that $p$ and $q$ are coprime and denote the set of all twist periodic orbits (non-twist periodic orbits) of $g$ of period $q$ and rotation number $p / q$ for $G$ by $T(G)(N(G)$ respectively).

LemmA 6. Let $G \in \mathscr{L}$ and assume that both $T(G)$ and $N(G)$ are non-empty. Then there exist points $X_{i}, Y_{i} \in \mathbb{R}(i=0,1, \ldots, q)$ such that

(i) $X_{i}<Y_{i}$ for $i=0,1, \ldots, q$;

(ii) $X_{q}=X_{0}+p$ and $Y_{q}=Y_{0}+p$;

(iii) $G\left(X_{i}\right)<X_{i+1}$ and $G\left(Y_{i}\right)>Y_{i+1}$ for $i=0,1, \ldots, q-1$;

(iv) if $j, k \in\{0,1, \ldots, q-1\}$ and $m, n \in \mathbb{Z}$ then: either

$$
Y_{j}+n<X_{k}+m \text { and } Y_{j+1}+n<X_{k+1}+m
$$

or

$$
Y_{k}+m<X_{j}+n \text { and } Y_{k+1}+m<X_{j+1}+n .
$$

Proof. Let $A \in T(G), B \in N(G)$. Take as $X_{0}^{\prime}$ and $Y_{0}^{\prime}$ two consecutive elements of $e^{-1}(A)$ and define $X_{i}^{\prime}=G^{i}\left(X_{0}^{\prime}\right), Y_{i}^{\prime}=G^{i}\left(Y_{0}^{\prime}\right)$ for $i=1,2, \ldots, q$. Then for $X_{i}^{\prime}, Y_{i}^{\prime}$ conditions (i) and (ii) hold, (iii) holds with equalities instead of inequalities, and (iv) holds with non-strict inequalities. Since the orbit $B$ has the same period and rotation number as $A$, but it is not twist, there are $j, k \in\{0,1, \ldots, q-1\}$ and points $Z \in\left(X_{j}^{\prime}, Y_{j}^{\prime}\right) \cap e^{-1}(B)$ and $T \in\left(X_{k}^{\prime}, Y_{k}^{\prime}\right) \cap e^{-1}(B)$ such that $G(Z)<X_{j+1}^{\prime}$ and $G(T)>Y_{k+1}^{\prime}$. We set

$$
X_{i}^{\prime \prime}=\left\{\begin{array}{ll}
X_{i}^{\prime} & \text { if } i \neq j \\
Z & \text { if } i=j
\end{array}, \quad Y_{i}^{\prime \prime}= \begin{cases}Y_{i}^{\prime} & \text { if } i \neq k \\
T & \text { if } i=k\end{cases}\right.
$$

for $i=0,1, \ldots, q-1$ and

$$
X_{q}^{\prime \prime}=X_{0}^{\prime \prime}+p, \quad Y_{q}^{\prime \prime}=Y_{0}^{\prime \prime}+p .
$$

Then for $X_{i}^{\prime \prime}, Y_{i}^{\prime \prime}$ conditions (i) and (ii) still hold, (iv) holds with non-strict inequalities, and instead of (iii) we have $G\left(X_{i}^{\prime \prime}\right) \leq X_{i+1}^{\prime \prime}, G\left(Y_{i}^{\prime \prime}\right) \geq Y_{i+1}^{\prime \prime}$ for $i=$ $0,1, \ldots, q-1$ and $G\left(X_{j}^{\prime \prime}\right)<X_{j+1}^{\prime \prime}, G\left(Y_{k}^{\prime \prime}\right)>Y_{k+1}^{\prime \prime}$. Thus, since $G$ is continuous, we can find $\varepsilon_{0}, \varepsilon_{1}, \ldots, \varepsilon_{q}>0$ such that $\varepsilon_{q}=\varepsilon_{0}$ and for the points $X_{i}=X_{i}^{\prime \prime}+\varepsilon_{i}$ and $Y_{i}=Y_{i}^{\prime \prime}-\varepsilon_{i}$ the conditions (i)-(iv) are satisfied.

Lemma 7. (a) The set of those $G \in \mathscr{L}$ for which there exist points $X_{i}, Y_{i}$ satisfying (i)-(iv), is open.

(b) If there exist points $X_{i}, Y_{i}$ satisfying (i)-(iv) for $G \in \mathscr{L}$ then $T(G)$ is non-empty. Proof. (a) If the conditions (i)-(iv) are satisfied for some $G \in \mathscr{L}$ and some $X_{i}, Y_{i}$, then clearly they are satisfied for all $F$ from some neighbourhood of $G$ for the same $X_{i}, Y_{i}$.

(b) By (iii) we have $G\left(\left[X_{i}, Y_{i}\right]\right) \supset\left[X_{i+1}, Y_{i+1}\right]$ for $i=0,1, \ldots, q-1$ and consequently there exists a point $Z \in\left[X_{0}, Y_{0}\right]$ such that $G^{i}(Z) \in\left[X_{i}, Y_{i}\right]$ for $i=$ $0,1, \ldots, q$ and $G^{q}(Z)=Z+p$. Hence, $e(Z)$ is a periodic point for $g$ of period $q$ and rotation number $p / q$. By (iv), its orbit is twist.

Lemma 8. The set of those $G \in \mathscr{L}$ for which $T(G)$ is non-empty, is closed. 
Proof. Let $G_{n} \rightarrow G$ as $n \rightarrow \infty$ and let $A_{n} \in T\left(G_{n}\right)$. Taking a subsequence if necessary, we may assume that we have $\lim _{n \rightarrow \infty} X_{n}=X$ for some $X_{n} \in e^{-1}\left(A_{n}\right)$ and $X \in \mathbb{R}$. Since $G_{n}^{q}\left(X_{n}\right)=X_{n}+p$ for each $n$, we obtain $G^{q}(X)=X+p$. Hence, $e(X)$ is a periodic point for $g$ and its rotation number is $p / q$. Since $p$ and $q$ are coprime, its period is $q$. By a continuity argument, $G$ restricted to the orbit of $X$ is non-decreasing, and since the period of $e(X)$ is $q$, it is strictly increasing. Hence the orbit of $e(X)$ is twist.

LeMMA 9. Each $F_{0} \in \mathscr{L}$ with an orbit $B \in N\left(F_{0}\right)$ may be joined by a curve $\left(F_{t}\right)_{0 \leq t \leq 1}$ in $\mathscr{L}$ to some $F_{1}$ with $T\left(F_{1}\right)$ non-empty in such a way that $\left.F_{t}\right|_{e^{-1}(B)}=\left.F_{0}\right|_{e^{-1}(B)}$ for all $t \in[0,1]$.

Proof. We can find $\alpha \in \mathbb{R}$ such that the set $\{(k / q)+\alpha\}_{k \in Z}$ is disjoint from $e^{-1}(B)$, and then deform $F_{0}$ continuously in $\mathscr{L}$ to $F_{1}$ (keeping $\left.F_{t}\right|_{e^{-1}(B)}$ unchanged) in such a way that

$$
F_{1}\left(\frac{k}{q}+\alpha\right)=\frac{k+p}{q}+\alpha \quad \text { for } k \in \mathbb{Z} .
$$

Proof of theorem A. (a) If $r / s \in \mathbb{Z}$ then we are done. If not, let $F$ be a lifting of $f$ such that $r / s$ is the right endpoint of $L_{F}(f)$. The proof follows similarly when $r / s$ is the left endpoint. Let $F^{\prime}=F-E(r / s)$. Then

$$
r^{\prime} / s=r / s-E(r / s) \in L_{F},(f) .
$$

Note that $\left(r^{\prime}, s\right)=1$ and $r^{\prime} / s \in(0,1)$. Then, by lemma 5 , all the periodic orbits of period $s$ and $F^{\prime}$-rotation number $r^{\prime} / s$ are TPO. Hence, these orbits are TPO of period $s$ and $F$-rotation number $r / s$.

(b) Suppose that $T(F)$ is empty. Then $N(F)$ is non-empty and we can take a curve $\left(F_{t}\right)_{0 \leq t \leq 1}$ with properties as in lemma 9 (here $F_{0}=F$ ). The set of those $t$ for which $T\left(F_{t}\right)$ is non-empty contains 1 by lemma 9 , is open by lemmas 6 and 7 (remember that $B \in N\left(F_{t}\right)$ for all $t$ ), and is closed by lemma 8 . Hence, $T\left(F_{0}\right)$ is non-empty - a contradiction.

\section{Topological entropy}

Let $p, q \in \mathbb{N}$ with $(p, q)=1$. There exist unique $k \in\{1, \ldots, q-1\}$ and $l \in$ $\{0,1, \ldots, p-1\}$ such that $k p-l q=1$. For each $i \in\{1, \ldots, p-1\}$ there exists a unique $j \in\{1, \ldots, q\}$ such that $j \equiv i k(\bmod q)$. We denote this $j$ by $\psi(p / q, i)$. This defines the function $\psi(p / q, \cdot):\{1, \ldots, p-1\} \rightarrow\{1, \ldots, q\}$. Note that if $p<q$ then it is one-toone.

For a rational number $p / q \neq 0$ with $(p, q)=1$, we define the polynomial

$$
H_{p / q}^{\prime}(z)=z^{q+1}-z^{q}-z-1-2 \sum_{i=1}^{|p|-1} z^{\psi(|p| / q, i)}
$$

We denote by $\alpha_{p / q}^{\prime}$ the largest root of $H_{p / q}^{\prime}$. Note that if $q=1$ then $\psi(|p| / q, i)=1$ for $i=1, \ldots,|p|-1$, and hence $H_{p}^{\prime}(z)=z^{2}-2|p| z-1$.

Proposition 10. Let $f \in C_{1}\left(S^{1}\right)$. If $0, p / q \in L(f)$ with $(p, q)=1$ and $p / q \neq 0$, then $h(f) \geq \log \alpha_{p / q}^{\prime}$. 
Proof. We recall that we are using the notation and the standard techniques of [2]. We divide the proof into four cases.

Case 1. Suppose $0<p<q$. From theorem A there exists a TPO of $f$ of period $q$ and rotation number $p / q$. Let $A$ be the set consisting of a fixed point $d$ with rotation number 0 and all elements of the above TPO, and $\bar{f}$ be the $A$-linearization of $f$. Let $G$ be the $A$-graph of $\bar{f}$. Since $G$ is a subgraph of the $A$-graph of $f$, from lemma 1.5 of [2] it follows that $h(f) \geq h(G)$. Now we are going to compute $h(G)$.

Let $F$ be a lifting of $\bar{f}$ such that $0 \in L_{F}(\bar{f})$ and $D$ be a fixed point of $F$ such that $e(D)=d$. The points of $e^{-1}(A)$ give us a partition of $[D, D+1]$ into intervals and we call these intervals from the left to the right $I_{1}, I_{2}, \ldots, I_{q+1}$. The map $F$ looks as in figure 5.

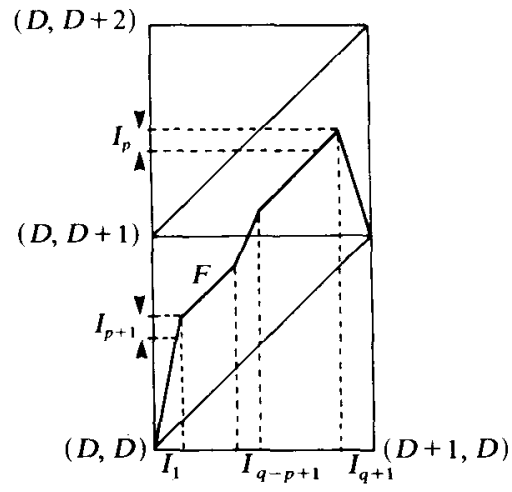

Figure 5

It is clear that $I_{1} \bar{f}$-covers $I_{j}$ with $j=1,2, \ldots, p+1 ; I_{q+1} \bar{f}$-covers $I_{j}$ with $j=$ $1,2, \ldots, p ; I_{q-p+1} \bar{f}$-covers $I_{1}$ and $I_{q+1}$, and $I_{j} \bar{f}$-covers $I_{j+p(\bmod q)}$ (when dealing with indices from the set $\{1, \ldots, q\}$, by $q(\bmod q)$ we mean $q$ instead of 0$)$ for $j \notin\{1$, $q-p+1, q+1\}$. Then $G$ has the following subgraph

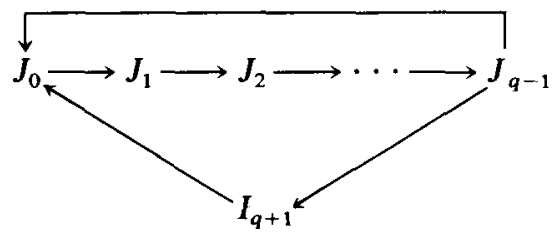

where $J_{j}=I_{j p+1(\bmod q)}$. Obviously $J_{0}=I_{1}$ and $J_{q-1}=I_{q-p+1}$. With this notation, we have that $J_{0}$ and $I_{q+1} \bar{f}$-cover, additionally, $J_{j}$ with $j \in B_{p / q}=\{j: 2 \leq j \leq q-1$ and $J_{j}=I_{k}$ with $\left.2 \leq k \leq p\right\}=\{j: 2 \leq j \leq q-1$ and $2 \leq j p+1(\bmod q) \leq p\}$. Furthermore, $J_{0}$ $\bar{f}$-covers itself. In such a way we obtain the whole graph $G$.

In order to compute $h(G)$ we take as a rome $R=\left\{R_{1}, R_{2}\right\}$ with $R_{1}=I_{1}$ and $R_{2}=I_{q+1}$. Now, we compute the elements $a_{i j}(z)=\sum_{t} w(t) z^{-l(t)}$ of the $2 \times 2$ matrix $A_{R}(z)$, where the summation is over all simple paths originating at $R_{i}$ and terminating at $R_{j}$, and $w(t)$ is the width of the simple path $t$ and $l(t)$ is its length. Then we 
define $V(z)=\sum_{j \in B_{p / q}} z^{j-q-1}$. Since the length of the paths

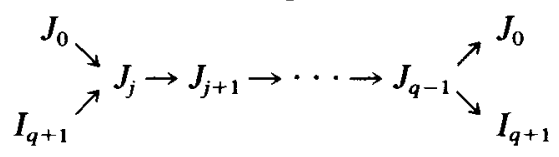

is $q-j+1$, we have $a_{11}(z)=z^{-1}+z^{-q}+V(z), \quad a_{12}(z)=z^{-q}+V(z), \quad a_{21}(z)=$ $z^{-1}+V(z), a_{22}(z)=V(z)$.

Now, from theorem 1.7 of [2] it follows that $h(G)$ is the logarithm of the largest root of the polynomial

$$
\begin{aligned}
z^{q+1} \operatorname{det}\left(A_{R}(z)-\mathrm{id}\right) & =z^{q+1}\left|\begin{array}{cc}
-2 & z^{-q}+1 \\
z^{-1}+1 & V(z)-1
\end{array}\right| \\
& =z^{q+1}-z^{q}-z-1-2 \sum_{j \in B_{p / q}} z^{j} .
\end{aligned}
$$

To finish the proof in case 1 it is enough to establish the following claim: the image of $\{1, \ldots, p-1\}$ under $\psi(p / q, \cdot)$ is $B_{p / q}$. Since both sets have cardinality $p-1$, it is sufficient to show that for each $i \in\{1, \ldots, p-1\}$ we have $\psi(p / q, i) \in B_{p / q}$, that is

$$
2 \leq \psi(p / q, i) p+1(\bmod q) \leq p .
$$

Since $\psi(p / q, i) \equiv i k(\bmod q)$, where $k p-l q=1$, we have $\psi(p / q, i)=n q+i k$ for some $n \in \mathbb{Z}$. Then

$$
\psi(p / q, i) p+1=(n q+i k) p+1=(n p+i l) q+i+1
$$

and hence

$$
\psi(p / q, i) p+1=i+1(\bmod q)
$$

This proves the claim.

Case 2. Suppose that $0<q<p$ with $q \neq 1$. In a similar way to case 1 , we have that $h(f) \geq h(G)$ where $G$ is the $A$-graph of a piecewise linear map $\bar{f}$ which has a lifting $F$ as in figure 6. Therefore $G$ is exactly the same as in case 1 adding the following arrows: $I_{1}$ and $I_{q+1} \bar{f}$-cover $m$ times $I_{j}$ for $j=1,2, \ldots, q+1$, where $1<p / q=m+r / q$ with $0<r \leq q$. We define

$$
W(z)=m \sum_{j=1}^{q} z^{-j}
$$

Then, as above, it follows that $h(G)$ is the logarithm of the largest root of the polynomial

$$
\begin{aligned}
z^{q+1} \operatorname{det}\left(A_{R}(z)-\mathrm{id}\right) & =z^{q+1}\left|\begin{array}{cc}
-2 & z^{-q}+1 \\
z^{-1}+1 & V(z)+W(z)-1
\end{array}\right| \\
& =z^{q+1}-z^{q}-z-1-2 m \sum_{j=1}^{q} z^{j}-2 \sum_{j \in B_{r / q}} z^{j} .
\end{aligned}
$$

We only need to check that each element of $\{1,2, \ldots, q\}$ is attained by $\psi(p / q, \cdot)$ either $m+1$ times if it is attained by $\psi(r / q, \cdot)$, or $m$ times if it is not attained by $\psi(r / q, \cdot)$. Take $k \in\{1, \ldots, q-1\}$ and $l^{\prime} \in\{0,1, \ldots, r-1\}$ such that $k r-l^{\prime} q=1$. Since $p=m q+r$, for $l=k m+l^{\prime}$ we have $0 \leq l^{\prime} \leq l \leq q m+r-1=p-1$ and $k p-l q=$ $k m q+k r-k m q-l^{\prime} q=1$. From the definition of $\psi$ it follows that if $i$ runs over the 


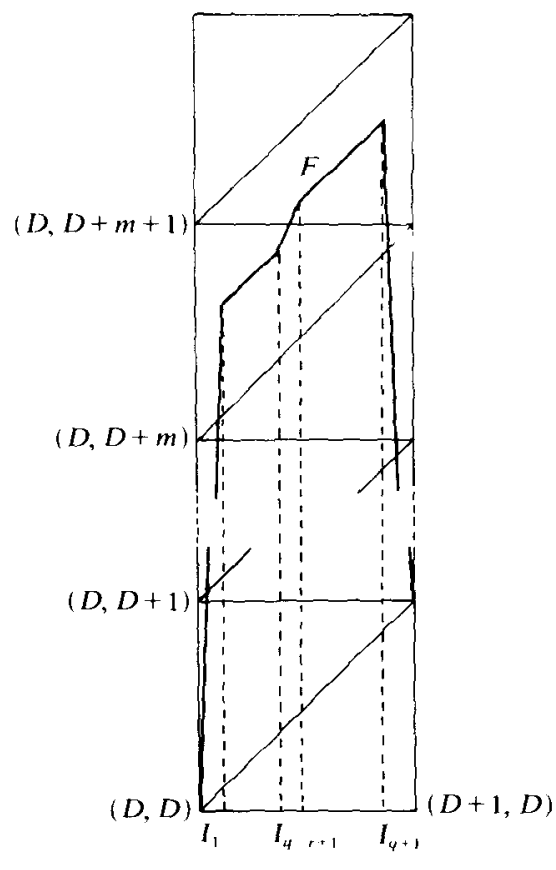

FIGURE 6

set $\{s q+1, s q+2, \ldots,(s+1) q\}$ (where $0 \leq s<m)$ then $\psi(p / q, i)$ runs over the set $\{1,2, \ldots, q\}$ and that for $i \in\{m q+1, m q+2, \ldots, m q+r-1\}$ we have $\psi(p / q, i)=$ $\psi(r / q, i-m q)$. This ends the proof of case 2 .

Case 3. Suppose that $p / q>0$ with $q=1$. Let $A$ be the set consisting of a fixed point with rotation number 0 and a fixed point with rotation number $p$. Let $\bar{f}$ be the $A$-linearization of $f$ and $F$ be a lifting of $\bar{f}$ for which 0 and $p$ are the rotation

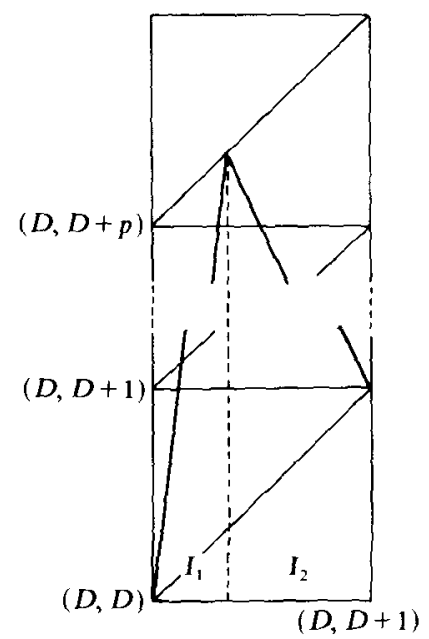

Figure 7 
numbers of these points. Let $D, D^{\prime} \in \mathbb{R}$ such that $F(D)=D$ and $F\left(D^{\prime}\right)=D^{\prime}+p$, with $D<D^{\prime}<D+1$. Then $F$ is as in figure 7. If $G$ is the $A$-graph of $\bar{f}$, then we have that $I_{1} \bar{f}$-covers $I_{1} p+1$ times and $\bar{f}$-covers $I_{2} p$ times, and $I_{2} \bar{f}$-covers $I_{1} p$ times and $\bar{f}$-covers $I_{2} p-1$ times, where $I_{1}=\left[D, D^{\prime}\right]$ and $I_{2}=\left[D^{\prime}, D+1\right]$. Now, if we take as a rome $R=\left\{I_{1}, I_{2}\right\}$, we have

$$
\begin{aligned}
z^{2} \operatorname{det}\left(A_{R}(z)-\mathrm{id}\right) & =z^{2}\left|\begin{array}{cc}
(p+1) z^{-1}-1 & p z^{-1} \\
p z^{-1} & (p-1) z^{-1}-1
\end{array}\right| \\
& =z^{2}-2 p z-1 .
\end{aligned}
$$

Case 4. Suppose $p / q<0$. By replacing $F(X)$ by $-F(-X)$, we obtain one of the cases $1,2,3$.

Proposition 11. Let $p / q \neq 0$ with $(p, q)=1$. Then there exists a map $f \in C_{1}\left(S^{1}\right)$ such that $L(f)=[0, p / q] \cap \mathbb{Q}(\operatorname{resp} . L(f)=[p / q, 0] \cap \mathbb{Q})$ if $p / q>0($ resp. $p / q<0)$ and $h(f)=\log \alpha_{p / q}^{\prime}$.

Proof. We consider the case $p / q>0$ and $q \neq 1$. For the other cases the proof is similar. Let $F: \mathbb{R} \rightarrow \mathbb{R}$ be the following continuous map:

(1) $F(0)=0, F(1)=1$;

(2) $F(X)=X+p / q$ for $X \in[p / 2 q, 1-p / 2 q]$;

(3) $F$ is linear on $[0, p / 2 q]$ and on $[1-p / 2 q, 1]$;

(4) $F(X+1)=F(X)+1$ for all $X \in \mathbb{R}$.

Let $f \in C_{1}\left(S^{1}\right)$ such that $F$ is a lifting of $f$. From [5] (see the proof of the theorem) it follows that $L(f)=[0, p / q] \cap \mathbb{Q}$. The graph $G$ given in cases 1 and 2 of proposition 10 is the $A$-graph of such an $f$, where $A$ is the partition of $S^{1}$ given by $e(\{0,1 / 2 q, 3 / 2 q, \ldots,(2 q-1) / 2 q\})$. Let $M$ be the transition matrix of $G$. It is well known (see [3, p. 250]) that the entropy of $f$ is equal to the entropy of the subshift of finite type with transition matrix $M$. By the computations made in the proof of proposition 10 , the largest eigenvalue of $M$ is equal to $\alpha_{p / q}^{\prime}$, and hence $h(f)=$ $\log \alpha_{p / q}^{\prime}$.

LEMMA 12. For $p, q \in \mathbb{N}$ with $(p, q)=1$ there exists a permutation $\sigma_{p / q}$ of the set $\{1, \ldots, p-1\}$ such that $\psi(p / q, i)=E\left(\sigma_{p / q}(i) q / p\right)+1$ for each $i \in\{1, \ldots, p-1\}$.

Proof. We take $k \in\{1, \ldots, q-1\}$ and $l \in\{0,1, \ldots, p-1\}$ such that $k p-l q=1$. Then $k=l q / p+1 / p$, and hence $i k=E(i l p / q)+1$ for each $i \in\{1, \ldots, p-1\}$. We set $\sigma_{p / q}(i)=l i(\bmod p)$ for $i \in\{1, \ldots, p-1\}$ (notice that $l=0$ only if $p=1$ ). Since $(l, p)=1, \sigma_{p / q}$ is a permutation of $\{1, \ldots, p-1\}$. We have $1<1+\sigma_{p / q}(i) q / p<q+1$ and $E(i l q / p) \equiv E\left(\sigma_{p / q}(i) q / p\right)(\bmod q)$. Therefore

$$
E\left(\sigma_{p / q}(i) q / p\right)+1=\psi(p / q, i) .
$$

From lemma 12 it follows immediately that $H_{p / q}^{\prime}=H_{p / q}$, and $\alpha_{p / q}^{\prime}=\alpha_{p / q}\left(H_{p / q}\right.$ and $\alpha_{p / q}$ were defined in the introduction).

LeMma 13. For $p, q \in \mathbb{N}$ and $z>1$ we have

$$
\sum_{j=1}^{p-1} z^{E(j q / p)+1}=\left(z^{q}-1\right) \sum_{n=0}^{\infty} z^{-E(n q / p)}-z^{q} .
$$


Proof. We have

$$
\begin{aligned}
\sum_{n=0}^{\infty} z^{-E(n q / p)} & =\sum_{k=0}^{\infty} \sum_{i=0}^{p-1} z^{-E((k p+i) q / p)} \\
& =\sum_{k=0}^{\infty} \sum_{i=0}^{p-1} z^{-E(i q / p)-k q}=\sum_{k=0}^{\infty} z^{-k q} \sum_{i=0}^{p-1} z^{-E(i q / p)},
\end{aligned}
$$

and hence

$$
\left(1-z^{-q}\right) \sum_{n=0}^{\infty} z^{-E(n q / p)}=\sum_{i=0}^{p-1} z^{-E(i q / p)}
$$

For $i \in\{1,2, \ldots, p-1\}$ the number $i q / p$ is not an integer, and hence $E(i q / p)+$ $E((p-i) q / p)+1=q$. Therefore, using lemma 12, we obtain

$$
\sum_{i=0}^{p-1} z^{-E(i q / p)}=1+\sum_{k=1}^{p-1} z^{E(k q / p)+1-q}=z^{-q} \sum_{j=1}^{p-1} z^{E(j q / p)+1}+1 .
$$

From this relation the lemma follows.

Recall that for $c \neq 0$ and $z>1$, we defined $Q_{c}(z)=z+1-2 \sum_{n=0}^{\infty} z^{-E(n /|c|)}$. Then, from lemma 13 it follows that:

LEMMA 14. For $p, q \in \mathbb{N}$ and $z>1$ we have $H_{p / q}(z)=\left(z^{q}-1\right) Q_{p / q}(z)$.

LEMma 15. For every $c>0$ the function $Q_{c}:(1, \infty) \rightarrow \mathbb{R}$ is strictly increasing.

Proof. For every $n \geq 0$ we have $-E(n /|c|) \leq 0$ and hence the function $z \mapsto z^{-E(n /|c|)}$ is non-decreasing. Since the function $z \mapsto z+1$ is strictly increasing, we obtain that $Q_{c}$ is strictly increasing.

LEMMA 16. For every $c \neq 0$ the equation $Q_{c}(z)=0$ has a unique root (which, of course, is larger than 1). If $c=p / q$ with $(p, q)=1$, then this root is equal to $\alpha_{p / q}$, the largest root of $H_{p / q}(z)$.

Proof. We have $\lim _{z \backslash 1} Q_{c}(z)=-\infty$ and $\lim _{z \rightarrow \infty} Q_{c}(z)=\infty$. Together with lemma 15, this implies that the equation $Q_{c}(z)=0$ has a unique root. Let $c=p / q$. Then, by lemma 14 the second part of the lemma follows.

For $c \neq 0$ we define $\beta_{c}$ as the unique root of the equation $Q_{c}(z)=0$. We have $\beta .: \mathbb{P} \backslash\{0\} \rightarrow(1, \infty)$ such that $\beta_{c}=\beta_{-c}$. Then, from now on we only study $Q_{c}$ and $\beta_{c}$ for $c>0$.

LEMMA 17. For every $z>1$ the function $Q .(z):(0, \infty) \rightarrow \mathbb{R}$ is strictly decreasing.

Proof. Let $0<c_{1}<c_{2}$. For every $n$ we have $-E\left(n / c_{2}\right) \geq-E\left(n / c_{1}\right)$ and for $n$ such that $1 / c_{2}<k / n \leq 1 / c_{1}$ for some integer $k$, the inequality is strict. Therefore $Q_{c_{2}}(z)<$ $Q_{c_{1}}(z)$ for all $z>1$.

From lemmas 15 and 17 it follows immediately:

LEMMA 18. The function $\beta .:(0, \infty) \rightarrow(1, \infty)$ is strictly increasing.

For $0<b, d$ set $k(b, d)=\min \{n \geq 0: E(n / d) \neq E(n / b)\}$. 
LEMMA 19. For every $y>1$, if $b$ and $d$ are bounded from above and $k(b, d) \rightarrow \infty$, then $\left|Q_{b}(z)-Q_{d}(z)\right| \rightarrow 0$ uniformly with respect to $z \in[y, \infty)$.

Proof. For $b, d<a$ and $z \geq y$ we have

$$
\begin{aligned}
\left|Q_{b}(z)-Q_{d}(z)\right| & =2\left|\sum_{n=0}^{\infty}\left(z^{-E(n / d)}-z^{-E(n / b)}\right)\right| \\
& \leq 4 \sum_{n=k(b, d)}^{\infty} z^{-E(n / a)} \\
& =4 z^{-k(b, d) / a} /\left(1-z^{-1 / a}\right) .
\end{aligned}
$$

For $k(b, d) \geq a$ we obtain

$$
\left|Q_{b}(z)-Q_{d}(z)\right| \leq 4 y^{-k(b, d) / a} /\left(1-y^{-1 / a}\right) .
$$

If $k(b, d) \rightarrow \infty$ then this tends to zero.

LEMMA 20. (a) For every $b>0$, if $d \nearrow b$ then $k(b, d) \rightarrow \infty$.

(b) For every irrational $b>0$, if $d \rightarrow b$, then $k(b, d) \rightarrow \infty$.

Proof. Take $n>0$. In both cases, if $d$ is sufficiently close to $b$, then there is no fraction with the denominator smaller than $n$ in $(1 / d, 1 / b]$ or $(1 / b, 1 / d]$ (depending on whether $b<d$ or $d<b)$. Then $E(m / d)=E(m / b)$ for all $m<n$. Consequently $k(b, d) \geq n$. Therefore, in both cases we obtain $k(b, d) \rightarrow \infty$.

LemMa 21. Let $c_{1}=p / q>0$ with $(p, q)=1$. Then

$$
Q_{c_{1}}(z)-\lim _{c \searrow c_{1}} Q_{c}(z)=2(z-1) /\left(z^{q}-1\right) .
$$

Proof. We have $\lim _{c \searrow c_{1}} E(n / c)=E\left(n / c_{1}\right)$ for all $n$ except for $n=k p$ with $k=1,2, \ldots$ For these exceptional $n, E\left(n / c_{1}\right)=k q$, but $\lim _{c \backslash c_{1}} E(n / c)=k q-1$. Therefore,

$$
Q_{c_{1}}(z)-\lim _{c \searrow c_{1}} Q_{c}(z)=2 \sum_{k=1}^{\infty}\left(z^{-k q+1}-z^{-k q}\right)=2(z-1) /\left(z^{q}-1\right) .
$$

From lemmas 14 and 21 it follows:

LEMMA 22. If $p, q \in \mathbb{N},(p, q)=1$, then the number $\lim _{c \backslash p / q} \beta_{c}$ is the largest root of the polynomial $H_{p / q}(z)-2 z+2$.

Since $H_{n}(z)=z^{2}-2 n z-1$ for all $n \in \mathbb{N}$, from lemmas 18 and 22 it follows:

LEMMA 23. For all $n \in \mathbb{N}$ we have $\beta_{c} \nearrow n+\left(n^{2}+1\right)^{\frac{1}{2}}$ and $\beta_{c} \searrow n+1+\left((n+1)^{2}-1\right)^{\frac{1}{2}}$, if $c \nearrow n$ and $c \searrow n$, respectively.

Proof of theorem C. This follows from lemmas 16, 14, 18, 15, 19, 20, 22 and 23 (to show that the jump of $\beta$. at $n$ belongs to $(2-(1 / n), 2-(1 / n+1))$, one can use the inequalities $n+(1 /(2 n+1))<\sqrt{n^{2}+1}<n+(1 / 2 n)$ and $n+1-(1 /(2 n+1))<$ $\sqrt{n^{2}+2 n}<n+1-(1 /(2 n+2))$.

Proof of theorem B. The first part follows immediately from proposition 10, lemma 12 and (b), (c) and (e) of theorem C. The second part follows from proposition 11, lemma 12 and (b) of theorem $C$. 
Remark 4. In the above proof of theorem B we use theorem A via proposition 10. But using lemma 5, (c) of theorem $C$ and arguments similar to proposition 10, we can prove theorem $B$ without the use of theorem $A$.

Remark 5. If we define $\beta_{0}=1$, then the map $\beta$. is continuous at zero. This means that if $L(f)=\{0\}$ then the contribution of the rotation interval to the topological entropy is zero.

This paper was made possible by an invitation of the Universitat Autònoma de Barcelona to M. Misiurewicz.

\section{REFERENCES}

[1] L. Block, E. Coven \& Z. Nitecki. Minimizing topological entropy for maps of the circle. Ergod. Th. \& Dynam. Sys. 1 (1981), 145-149.

[2] L. Block, J. Guckenheimer, M. Misiurewicz \& L. S. Young. Periodic points and topological entropy of one dimensional maps. Springer Lect. Notes in Math. 819 (1980), 18-39.

[3] M. Denker, C. Grillenberger \& K. Sigmund. Ergodic Theory on Compact Spaces. Lect. Notes in Math. 527. Springer, 1976.

[4] L. Jonker \& D. Rand. The periodic orbits and entropy of certain maps of the unit interval. J. London Math. Soc. 22 (1980), 175-181.

[5] M. Misiurewicz. Periodic points of maps of degree one of a circle, Ergod. Th. \& Dynam. Sys. 2 (1982), 221-227.

[6] M. Misiurewicz. Twist sets for maps of the circle. Preprint, Warsaw, 1983. 